Review

\title{
Effects of Different Components of Yoga: A Meta-Synthesis
}

\author{
Karin Matko ${ }^{1,}{ }^{*}$, Holger C. Bringmann ${ }^{2,3}$, Peter Sedlmeier ${ }^{1}$
}

1. Chemnitz University of Technology, Chemnitz, Germany; E-Mails: karin.matko@psychologie.tuchemnitz.de; peter.sedlmeier@psychologie.tu-chemnitz.de

2. Institute for Social Medicine, Epidemiology and Health Economics, Charité-Universitätsmedizin, corporate member of Freie Universität Berlin, Humboldt-Universität zu Berlin, and Berlin Institute of Health, Berlin, Germany; E-Mail: holger.bringmann@charite.de

3. Department of Psychiatry, Psychosomatics and Psychotherapy, Diakoniekliniken Zschadrass, Colditz, Germany

* Correspondence: Karin Matko; E-Mail: karin.matko@psychologie.tu-chemnitz.de

Academic Editor: Viann N. Nguyen-Feng

Special Issue: Yoga and Mindfulness

OBM Integrative and Complementary Medicine 2021, volume 6 , issue 3

doi:10.21926/obm.icm.2103030
Received: March 08, 2021

Accepted: August 30, 2021

Published: September 13, 2021

\begin{abstract}
Traditional yoga encompasses a variety of practices, such as postures, breathing techniques, meditation, and ethical teachings. However, little is known about how different components of yoga contribute to its overall effect. In this meta-synthesis, we comprehensively summarized the current evidence on differential and incremental effects of various yoga components collected from available meta-analyses. For this meta-analysis, we searched Medline/PubMed, Scopus, PsychInfo, and the Cochrane Library in July 2021. We selected only meta-analyses that performed subgroup analyses comparing the effects of different yoga components. There were no restrictions regarding yoga type, study population, or outcome variables. Nineteen meta-analyses were identified that evaluated a multitude of variables. These meta-analyses varied greatly with regard to population, study design, and yoga components under investigation. However, combined interventions incorporating multiple components consistently outperformed simple interventions. In this regard, adding breathing
\end{abstract}

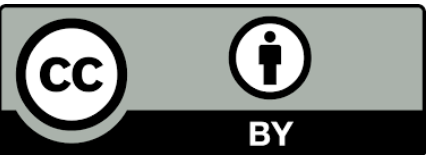

(C) 2021 by the author. This is an open access article distributed under the conditions of the Creative Commons by Attribution License, which permits unrestricted use, distribution, and reproduction in any medium or format, provided the original work is correctly cited. 
and/or meditation practices to yoga interventions proved particularly beneficial. However, specific components or combinations were more effective in enhancing certain variables or clinical conditions, suggesting a need for customized programs. Comparable multi-component mind-body interventions, such as yoga and mindfulness-based stress reduction, were often equally efficient. Nevertheless, most findings are preliminary, and certain components such as the ethical part of yoga are particularly under-researched.

Conclusion: Future studies should isolate and compare all components of yoga and evaluate additive effects by investigating their different combinations. The researchers should refer to theoretical frameworks, use rigorous methodology, and consider individual factors.

\section{Keywords}

Yoga components; mind-body interventions; postures; breathing practices; meditation; ethics

\section{Introduction}

With an increasing number of people practicing yoga, it has emerged as one of the most widely practiced mind-body interventions in the West [1, 2]. It is commonly used for preventing stress and relaxing the body and mind $[3,4]$. An increasing number of studies are acknowledging the positive effects of yoga on alleviating stress [5, 6] and promoting mental and physical health [7-9]. However, yoga encompasses a variety of practices, and it is unclear how these practices contribute to the overall effect of yoga. The ancient discipline of yoga originated in India and includes diverse practices such as ethical teachings (yamas and niyamas), physical postures (asana), breathing techniques (pranayama), and meditation techniques (pratyahara, dharana, dhyana, and samadhi) $[10,11]$. Research has shown that these practices can produce considerably different effects [1214]. Conversely, comparative and in-depth studies into the differential and incremental effects of yoga components are scarce. Furthermore, previous research often suffered from poor methodological quality and high heterogeneity among yoga interventions. Thus, more studies are warranted to evaluate and disentangle the effects of different components of yoga [15-18].

Recent theoretical proposals have delineated possible working mechanisms of various yoga components. For instance, Gard et al. [15] proposed an integrative framework and neurophysiological network model of yoga that focused on the enhancement of top-down and bottom-up self-regulatory mechanisms. The authors posited that yoga can be broken down into a skillset of four major tools, namely, ethics, postures, breath regulation, and meditation. All four tools contribute to optimizing self-regulation and developing an adaptive stress response. In their model, ethical beliefs promote the top-down initiation, monitoring, and maintenance of behavioral change. In contrast, postures, breath regulation, and meditation elicit both top-down and, particularly, bottom-up processes. The sustained practice of meditation and "meditation in motion" during posture and breath practices facilitates top-down attention control, which eventually supports behavioral change. In addition, the aspect of the embodiment in these practices enhances the bottom-up processes of sensory awareness, viscerosomatic integration, and parasympathetic control. With continued practice, these regulatory pathways are believed to become more 
automatized, leading to increased cognitive, emotional, and behavioral regulation and psychological and physical well-being. In the context of yoga components, meditation often refers to sitting meditation, referring to Patanjali's eight limbs, and distinguishing it from yoga postures [11]. However, yoga postures are sometimes described as a meditative movement [15]. Current research has delineated meditation with movement as a rightful category of meditation in itself [19]. Yet, in this synthesis, we will conform with the traditional interpretation of meditation as sitting meditation.

Two other proposals have described yoga as either a modern psychophysiological therapeutic practice consisting of movement-, breath-, and attention-based techniques [18] or an ethically grounded, transformational process of exploring values, causes of suffering, and eudaemonic wellbeing [20]. Schmalzl et al. [18] outlined the bottom-up neurophysiological and top-down neurocognitive processes affected by yoga movement, breath, and attention practices, independent of the traditional or ethical background. In contrast, Sullivan et al. [20] delineated how the ethical first-person inquiry through yamas and niyamas leads to an intentional reorientation of the identity, meaning, and purpose in life. Postures, breath regulation, and meditation can support the shifting from the experience of suffering to the experience of eudaemonic well-being.

These proposals strongly encourage the empirical investigation and systematic evaluation of the hypothesized links and mechanisms. These specifically suggest conducting longitudinal, comparative, or dismantling studies. However, the heterogeneity of yoga styles and the multitude of possible "active ingredients" in yoga and their manifold interactions make the investigation of its components challenging. Modern styles of yoga vary considerably with respect to the level of emphasis placed on various components. Although certain styles primarily focus on postures and breathing practices (e.g., lyengar, Hatha), others comprise mainly breathing practices (e.g., Sudarshan Kriya) or meditation (e.g., Sahaja). Some styles include mantras and chanting (e.g., Kundalini), whereas others integrate and synthesize as many aspects and components as possible (e.g., Kripalu). Almost all styles end their yoga practice with a period of deep relaxation in a supine position (shavasana). McCrary provides an overview of different yoga styles [21]. Scientific yoga interventions mirror this diversity of styles, making it hard to reliably conclude about the relative effects or importance of each yoga component.

\subsection{Evidence from Comparative Studies}

Previous yoga studies have evaluated and partially compared postures, breathing techniques, relaxation, chanting, meditation, and ethical education. These studies vary greatly in the degree of specificity and disassembling of components and practices under investigation. Certain studies compared, for example, very specific breathing practices to each other $[22,23]$, whereas others compared rather complex yoga interventions to meditation or relaxation techniques [24, 25] or stress management programs [26, 27]. Still, other studies more specifically examined the inclusion/exclusion of specific yoga components or different combinations of postures, breathing practice, and/or meditation [12, 14, 28-30] with often inconclusive results. The majority of studies investigated the effects of more or less intense yoga interventions of mostly 6 to 12 weeks, although certain studies compared single sessions of different yoga components [31, 32]. Furthermore, there existed variations regarding outcome variables, the intensity of treatments, methodological quality, sample size, and population, undermining the possibility of generalizing their findings. 
Interestingly, a review of the available comparative studies revealed that combined interventions seemed more effective than simple interventions. Comparative treatments in a given study were often equal in length. Nonetheless, outcomes were better for treatments combining several elements of yoga practice [14, 27, 29, 31, 33-40]. Combined treatments improved depression, anxiety, mood, sleep latency, interoceptive awareness, mindfulness, emotion and self-regulation, and physiological markers of stress.

However, findings were not always consistent, indicating a mixed state of affairs. Certain studies reported no difference between yoga interventions and progressive muscle relaxation [25], stress management [26, 41], or different forms of (mindfulness) meditation [42, 43] on similar outcome variables. In these cases, the effect was the same in both simple and combined interventions. Additionally, a multi-component mindfulness intervention exerted a greater effect on working memory capacity than a multi-component yoga intervention [24]. Interestingly, in the latter study, stress and anxiety scores improved for all conditions, including the waitlist control. Thus, this amelioration could not be attributed to the "active ingredients" of the two treatments. Nonetheless, a comparison of simple meditation treatments to multi-component yoga interventions showed that the latter was more effective in improving obsessive-compulsive pathology and mood [37, 38]. Another study reported that a combination of yoga and physical exercise outperformed both single treatments in enhancing well-being and reducing anxiety and stress [44]. Yet, in this study, this could be ascribed to higher dosage and exercise time in the combined treatment. Interestingly, customized treatments such as cognitive-behavioral therapy for a generalized anxiety disorder or self-hypnosis for cancer patients were more effective than combined yoga treatments $[45,46]$.

Frequently, the combined yoga treatments mentioned above included certain forms of yoga postures or breathing techniques; the latter appeared to be particularly relevant. For example, Bhavanani et al. [47] found that yoga breathing practice was more effective than yoga postures in enhancing reaction to visual stimuli. In addition, breathing practice helped dental students stay calm during their first surgical procedure more than a short stress-management lecture [36]. Schmalzl et al. [30] compared a movement-based yoga intervention to a breathing-based yoga intervention and found that both treatments reduced stress and salivary cortisol; however, sustained attention only improved in the breathing-based intervention. Similarly, incorporating a breathing practice into a combined yoga treatment was more effective in reducing stress and increasing mindfulness than including a visualization meditation technique [28]. In contrast, Vasanthan et al. [48] found that 6 months of either yoga postures or breathing practices equally improved cardiovascular autonomic functions. Yoga involves diverse breathing techniques with considerably different, and sometimes even opposing, effects on practitioners [22, 23]. Brown and Gerbarg [49] studied different breathing techniques and their psychological, physiological, and neurological effects and proposed an integrative neurophysiologic model.

Previous studies compared different treatments to each other, such as multi-component yoga programs and stress-reduction or mindfulness programs. Only a few truly contrasted isolated components of yoga practice, such as yoga postures versus breathing practice $[14,47,48]$, or supine meditation versus supine relaxation [50], with often inconclusive results. A few studies used an incremental design to compare the same intervention but removed or added a specific component to it. In most cases, the more complex treatment was superior to the reduced one [34, 35, 39]. Two of these studies explicitly examined the effects of adding an ethical education component to a yoga or meditation intervention. Smith et al. [39] reported a higher efficiency of the ethical yoga 
intervention in decreasing anxiety and salivary cortisol. Similarly, Matko et al. [34] reported that ethical education significantly enhanced the well-being of participants. According to traditional yoga experts, yoga should be practiced in its entirety, including its ethical aspects [51]. Similarly, a panel of experienced yoga teachers deemed the cultivation of positive values, attitudes, and behaviors important or essential for alleviating mental health conditions [52]. Thus, research findings are equivocal and more studies are required to evaluate yoga in its completeness and disentangle the specific effects of yoga ethics, postures, breathing practices, and meditation. In addition, each component of yoga interventions should be accurately described to facilitate the comparison between studies using, for example, the specifically developed Essential Properties of Yoga Questionnaire (EPYQ; [53]).

\subsection{The Present Meta-Synthesis}

Numerous meta-analyses and reviews have in the past attempted to synthesize the vast number of yoga studies; however, these suffered from heterogeneity and the lack of methodological rigor. Meta-analyses overcome the limitations of single studies as they aggregate the effects of multiple studies, thereby allowing more robust statements concerning the effects of different yoga components. However, only a few meta-analyses explicitly conducted subgroup analyses of studies with varying yoga components. These summaries are of particular relevance to understanding yoga practice as a whole as well as for the development of interventions tailored to the needs of specific clinical and non-clinical populations $[17,18]$.

This meta-synthesis summarizes the current evidence on the differential and incremental effects of various yoga components. We conducted a systematic review of all available meta-analyses that have conducted subgroup analyses based on the inclusion or exclusion of specific yoga components. This process was based on the theoretical framework proposed by Gard et al. [15], which delineates yoga postures, breathing practices, meditation, and ethics as the main components of yoga practice. In addition, we included meta-analyses that compared studies on yoga and studies on mindfulnessbased interventions, such as mindfulness-based stress reduction (MBSR; [54]) or its derivatives. MBSR is one of the most researched mind-body interventions to date. It combines mindfulness meditation and certain components of Buddhist practice with yoga postures and movement practices. Thus, its multi-component structure is comparable to that of traditional yoga. Lately, several attempts have been made to dismantle and evaluate single components of MBSR interventions. Two studies asked participants to log their respective daily practice times of each component. In one study [55], yoga practice time was more strongly related to improvements in mindfulness, perceived stress, anxiety, and psychological well-being than formal sitting meditation. Gallegos et al. [56] found that both yoga practice and sitting meditation time were associated with a higher posttreatment insulin-like growth factor; however, only yoga significantly increased positive affect. Other studies evaluated different components of MBSR by comparing active treatment groups and reported differential effects of yoga postures and diverse meditation techniques $[13,57-60]$. In two of these studies, the yoga component reduced stress or increased well-being more than the meditative components $[13,58]$. Therefore, it appeared promising to compare and evaluate the components of mind-body interventions that shared similar practices, such as yoga and mindfulness-based interventions. With this approach, we aim to shed light on the 
undeniable diversity in yoga research and support future research efforts on evaluating yoga components.

\section{Methods}

\subsection{Literature Search}

The meta-synthesis was planned and conducted in accordance with the recommended procedures for research syntheses [61]. A systematic literature search was performed in July 2021 using the databases Medline/PubMed, Scopus, PsychINFO, and the Cochrane Library. The following search strategy was used for all databases: "Yoga" [Title] AND "Meta-analysis" [Title]). After removing the duplicate entries, the search yielded 125 potentially relevant meta-analyses (Figure 1). Next, protocols, comments, corrections, and reprints of meta-analyses were excluded, and 108 full-text meta-analyses were assessed for eligibility.

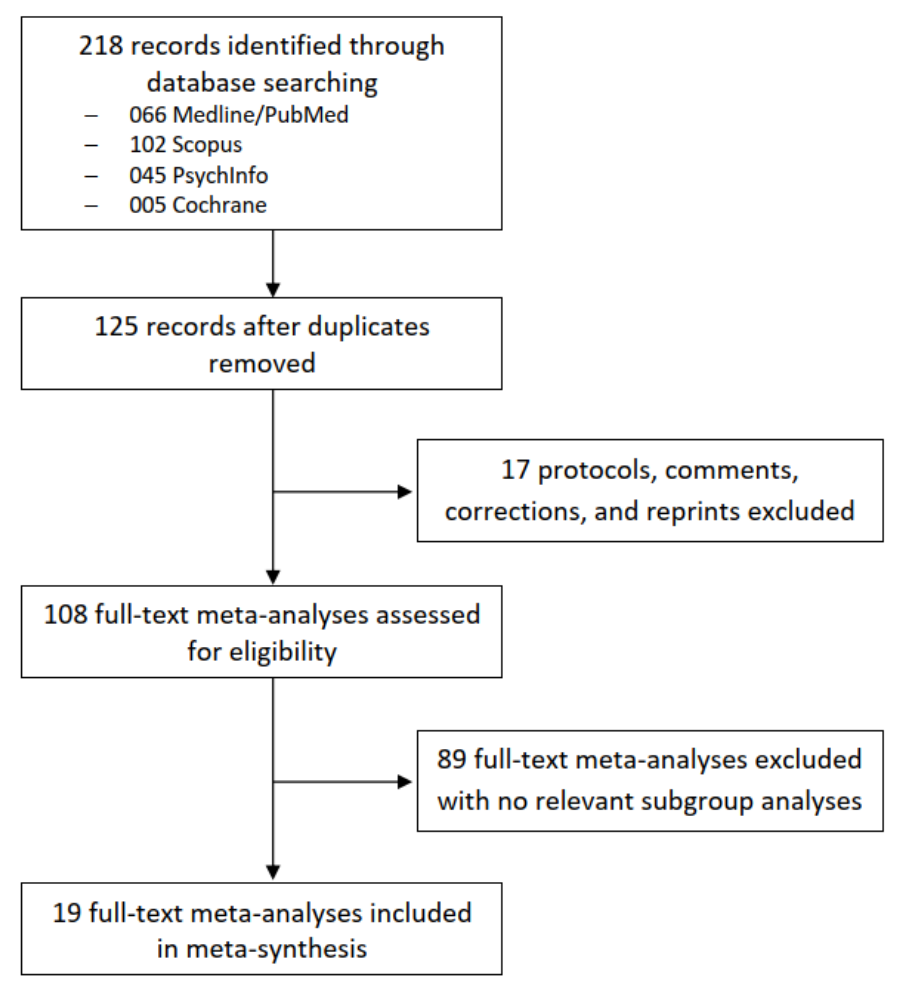

Figure 1 Flow diagram showing the search strategy.

\subsection{Eligibility Criteria}

All meta-analyses on the effects of yoga were considered eligible if they conducted relevant subgroup analyses that compared different yoga components. These yoga components could be postures/asana, breathing practice/pranayama, meditation/dhyana/mindfulness, education/ethics/philosophy/yamas/niyamas. Meta-analyses contrasting yoga and mindfulnessbased approaches were eligible when they differentiated both types of practice in the subgroup analyses. There were no restrictions regarding the yoga type, study population, or outcome variables. 


\subsection{Synthesis of Results}

Nineteen meta-analyses were examined and synthesized descriptively. The first author (KM) read all meta-analyses and extracted the study population, the number and designs of the included studies, the major effects reported in each meta-analysis, and the results of relevant subgroup analyses. Only subgroup analyses related to the differential effects of different yoga components were included in this overview. Next, KM deduced a primary conclusion for each meta-analysis in dialogue with all coauthors. Finally, the results of this systematic analysis were summarized and evaluated narratively.

\section{Results}

Table 1 provides an overview of all 19 meta-analyses summarized in this synthesis. These vary with regard to the outcome variables, population, sample size, study design of included studies, control groups, and yoga components under investigation in the subgroup analyses. The effect sizes of subgroup analyses that yielded significant differences are given in parentheses in the form of (with component vs. without component) if they were reported in the respective meta-analyses. The effect sizes that were not reported are marked as such (n.r.). 
Table 1 Results of previous meta-analyses that compared different components of yoga.

\begin{tabular}{|c|c|c|c|c|c|}
\hline Meta-analysis & Population/outcome & Sample & Mean effects & Subgroup analyses & Conclusion \\
\hline $\begin{array}{l}\text { Breedvelt et al. } \\
\text { [5] }\end{array}$ & $\begin{array}{l}\text { College } \\
\text { students/depression, } \\
\text { anxiety, stress }\end{array}$ & $\begin{array}{l}23 \text { RCTs } \\
(n=1,373)\end{array}$ & $\begin{array}{l}\text { Yoga, meditation, mindfulness, } \\
\text { and MBSR interventions } \\
g=0.61 \text { (overall) } \\
g=0.42 \text { (depression) } \\
g=0.46 \text { (anxiety) } \\
g=0.42 \text { (stress) } \\
g=0.61 \text { (overall pooled effect) } \\
g=0.52 \text { (waitlist control) } \\
g=0.39 \text { (no treatment) } \\
g=0.13 \text { (active control, n.s.) }\end{array}$ & $\begin{array}{l}\text { No differences between } \\
\text { yoga, meditation, } \\
\text { mindfulness, and MBSR } \\
\text { interventions }\end{array}$ & $\begin{array}{l}\text { No differences } \\
\text { between } \\
\text { combined } \\
\text { interventions }\end{array}$ \\
\hline $\begin{array}{l}\text { Chimiklis et al. } \\
\text { [62] }\end{array}$ & $\begin{array}{l}\text { Youth with ADHD/ } \\
\text { ADHD symptoms, } \\
\text { executive function } \\
\text { deficits, social } \\
\text { functioning }\end{array}$ & $\begin{array}{l}11 \text { studies } \\
(n=251)\end{array}$ & $\begin{array}{l}\text { Yoga, meditation, and } \\
\text { mindfulness-based interventions } \\
\text { ADHD symptoms: } \\
g=0.57 \text { (parent reported) } / g= \\
0.23 \text { (teacher reported) } \\
\text { Inattention and attention } \\
\text { problems: } \\
g=0.35 / g=0.31 \\
\text { Hyperactivity: } \\
g=0.39 / g=0.22 \\
\text { On-task behavior: } \\
g=1.22 \text { (researcher reported) } \\
\text { Executive function (teacher } \\
\text { report): } \\
g=0.31 \text { (metacognitive; } \text { n.s.) }\end{array}$ & $\begin{array}{l}\text { Mindfulness-based } \\
\text { interventions (e.g., MBSR, } \\
\text { MBCT) had a greater effect } \\
\text { on hyperactivity than yoga } \\
\text { interventions alone or } \\
\text { combined yoga plus } \\
\text { meditation interventions } \\
\text { (n.r.); other measures } \\
\text { improved across all types of } \\
\text { interventions }\end{array}$ & $\begin{array}{l}\text { Combined } \\
\text { intervention is } \\
\text { more effective }\end{array}$ \\
\hline
\end{tabular}




\begin{tabular}{|c|c|c|c|c|c|}
\hline Meta-analysis & Population/outcome & Sample & Mean effects & Subgroup analyses & Conclusion \\
\hline & & & $\begin{array}{l}g=0.67 \text { (behavioral) } \\
\text { Child-parent relationship: } \\
g=0.50 \text { (child reported) } \\
\text { Parent mindfulness: } \\
g=0.31 \text { (n.s.) } \\
\text { Parent stress: } \\
g=0.44\end{array}$ & & \\
\hline Cramer et al. [63] & $\begin{array}{l}\text { COPD/ } \\
\text { Quality of life, } \\
\text { dyspnea, exercise } \\
\text { capacity, pulmonary } \\
\text { function }\end{array}$ & $\begin{array}{l}11 \text { RCTs } \\
(n=586)\end{array}$ & $\begin{array}{l}\text { Yoga compared to no treatment: } \\
S M D=0.57 \text { (quality of life, n.s.) } \\
S M D=-0.23 \text { (dyspnea, n.s.) } \\
S M D=0.54 \text { (exercise capacity) } \\
S M D=0.47 \text { (forced expiratory } \\
\text { volume in one second, n.s.) } \\
\text { Positive effects on the quality of } \\
\text { life, exercise capacity, and } \\
\text { predicted forced expiratory } \\
\text { volume in } 1 \text { s when analyses } \\
\text { were limited to studies using the } \\
\text { same COPD assessment test. }\end{array}$ & $\begin{array}{l}\text { Breathing-based yoga } \\
\text { interventions had positive } \\
\text { effects on dyspnea (SMD = - } \\
0.43 \text { ), exercise capacity (MD } \\
=22.74) \text {, and predicted } \\
\text { forced expiratory volume in } \\
1 \mathrm{~s}(M D=3.97 \text { ) compared to } \\
\text { no treatment; complex yoga } \\
\text { interventions had positive } \\
\text { effects only on exercise } \\
\text { capacity (n.r.). }\end{array}$ & $\begin{array}{l}\text { Breathing is more } \\
\text { effective than } \\
\text { other } \\
\text { combinations }\end{array}$ \\
\hline Cramer et al. [64] & $\begin{array}{l}\text { Hypertension/blood } \\
\text { pressure }\end{array}$ & $\begin{array}{l}7 \text { RCTs } \\
(n=452)\end{array}$ & $\begin{array}{l}\text { Yoga compared to usual care: } \\
M D=-9.65 \text { (systolic BP) } \\
M D=-7.22 \text { (diastolic BP) } \\
\text { Yoga compared to exercise: } \\
\text { No difference }\end{array}$ & $\begin{array}{l}\text { One breathing-based yoga } \\
\text { intervention that did not } \\
\text { include postures (without } \mathrm{P} \text { ) } \\
\text { was more effective (systolic: } \\
M D=-6.56 \text {; diastolic: } M D= \\
-3.42 \text { ) than interventions } \\
\text { that did (with } \mathrm{P} \text { ) ( } M D=- \\
10.43 \text { n.s.; } M D=-8.20 \text { n.s.) }\end{array}$ & $\begin{array}{l}\text { Breathing is more } \\
\text { effective than } \\
\text { postures. }\end{array}$ \\
\hline
\end{tabular}




\begin{tabular}{|c|c|c|c|c|c|}
\hline Meta-analysis & Population/outcome & Sample & Mean effects & Subgroup analyses & Conclusion \\
\hline Cramer et al. [65] & $\begin{array}{l}\text { Chronic neck pain/pain } \\
\text { intensity, pain-related } \\
\text { disability, quality of } \\
\text { life, mood }\end{array}$ & $\begin{array}{l}3 \text { RCTs } \\
(n=188)\end{array}$ & $\begin{array}{l}\text { Yoga compared to usual care: } \\
S M D=-1.28 \text { (pain intensity) } \\
S M D=-0.97 \text { (pain-related } \\
\text { disability) } \\
S M D=0.57 \text { (quality of life) } \\
S M D=-1.02 \text { (mood) }\end{array}$ & $\begin{array}{l}\text { No differences in effects } \\
\text { between exercise-based } \\
\text { (with P) and meditation- } \\
\text { based (without P) yoga } \\
\text { interventions. }\end{array}$ & $\begin{array}{l}\text { No difference } \\
\text { between yoga } \\
\text { postures and } \\
\text { meditation. }\end{array}$ \\
\hline Cramer et al. [66] & $\begin{array}{l}\text { Depressive disorder or } \\
\text { elevated levels of } \\
\text { depression/depression, } \\
\text { anxiety }\end{array}$ & $\begin{array}{l}12 \text { RCTs } \\
(n=619)\end{array}$ & $\begin{array}{l}\text { Yoga compared to usual care: } \\
S M D=-0.69 \text { (depression) } \\
S M D=-0.00 \text { (anxiety, n.s.) } \\
\text { Yoga compared to relaxation: } \\
S M D=-0.39 \text { (depression) } \\
S M D=-0.79 \text { (anxiety) } \\
\text { Yoga compared to aerobic } \\
\text { exercise: } \\
S M D=-0.59 \text { (depression) }\end{array}$ & $\begin{array}{l}\text { Yoga interventions based on } \\
\text { meditation and/or } \\
\text { breathing (without P) were } \\
\text { more effective for reducing } \\
\text { depressive symptoms (SMD } \\
=-0.62 \text { ) and anxiety (SMD = } \\
-0.79 \text { ) than exercise-based } \\
\text { (SMD = -0.36 n.s.; SMD =- } \\
0.00 \text { n.s.) or combined yoga } \\
\text { interventions (with } p \text { ) (SMD } \\
=-0.42 \text { n.s.; anxiety n.r.). }\end{array}$ & $\begin{array}{l}\text { Meditation } \\
\text { and/or breathing } \\
\text { are more effective } \\
\text { than postures. }\end{array}$ \\
\hline Cramer et al. [67] & $\begin{array}{l}\text { Asthma/asthma } \\
\text { control, symptoms, } \\
\text { quality of life, } \\
\text { pulmonary function }\end{array}$ & $\begin{array}{l}14 \text { RCTs } \\
(n=824)\end{array}$ & $\begin{array}{l}\text { Yoga compared to usual care: } \\
\text { Risk ratio = } 10.64 \text { (asthma } \\
\text { control) } \\
S M D=-0.37 \text { (asthma symptoms) } \\
S M D=0.86 \text { (quality of life) } \\
S M D=0.49 \text { (peak expiratory } \\
\text { flow) } \\
\text { Yoga compared to psychological } \\
\text { interventions: } \\
S M D=0.61 \text { (quality of life) }\end{array}$ & $\begin{array}{l}\text { Combined ( } P, B, M) \text { and } \\
\text { breathing-based yoga } \\
\text { interventions were similarly } \\
\text { effective, whereas } \\
\text { meditation-based yoga was } \\
\text { not effective (n.r.). }\end{array}$ & $\begin{array}{l}\text { Combined } \\
\text { interventions } \\
\text { (especially } \\
\text { breathing) are } \\
\text { more effective. }\end{array}$ \\
\hline
\end{tabular}




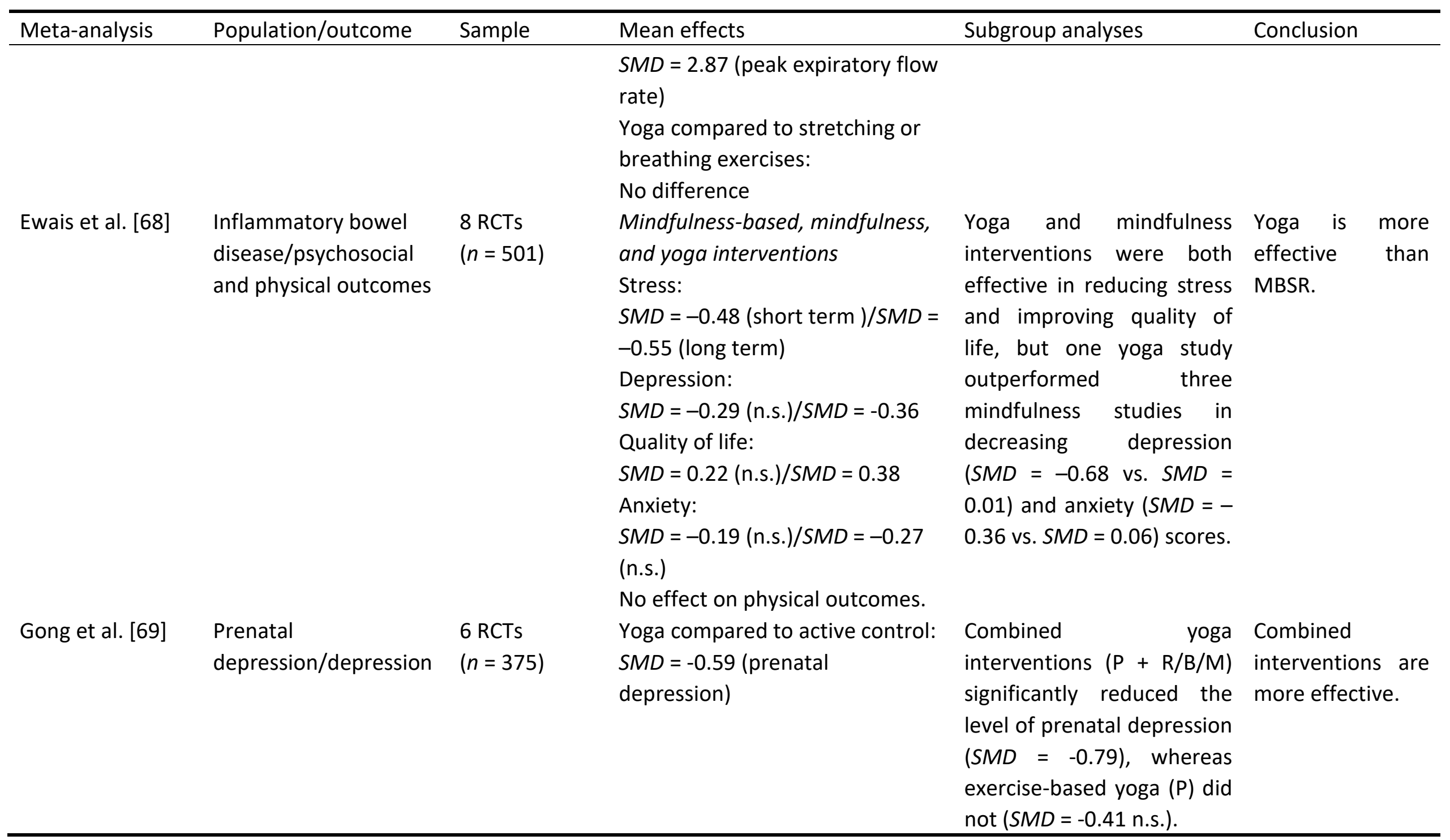




\begin{tabular}{|c|c|c|c|c|c|}
\hline Meta-analysis & Population/outcome & Sample & Mean effects & Subgroup analyses & Conclusion \\
\hline $\begin{array}{l}\text { Gothe \& McAuley } \\
{[70]}\end{array}$ & $\begin{array}{l}\text { Any } \\
\text { population/cognitive } \\
\text { function }\end{array}$ & $\begin{array}{l}15 \text { RCTs and } 7 \\
\text { repeated- } \\
\text { measures } \\
\text { studies } \\
(n=1,894)\end{array}$ & $\begin{array}{l}\text { RCTs: } \\
g=0.33 \text { (overall cognitive } \\
\text { function) } \\
g=0.29 \text { (attention, processing } \\
\text { speed) } \\
g=0.27 \text { (executive function) } \\
g=0.18 \text { (memory, n.s.) } \\
\text { Repeated measures (acute } \\
\text { effects): } \\
g=0.58 \text { (overall cognitive } \\
\text { function) } \\
g=0.49 \text { (attention, processing } \\
\text { speed) } \\
g=0.39 \text { (executive function) } \\
g=0.78 \text { (memory) }\end{array}$ & $\begin{array}{l}\text { Breathing and meditative } \\
\text { exercises, but not physical } \\
\text { exercises, were consistently } \\
\text { associated with improved } \\
\text { cognitive performance } \\
\text { (n.r.). }\end{array}$ & $\begin{array}{l}\text { Meditation and } \\
\text { breathing are } \\
\text { more effective } \\
\text { than postures. }\end{array}$ \\
\hline Hagins et al. [71] & $\begin{array}{l}\text { Hypertension/blood } \\
\text { pressure }\end{array}$ & $\begin{array}{l}17 \text { controlled } \\
\text { studies } \\
(n=1,013)\end{array}$ & $\begin{array}{l}\text { Overall effect: } \\
M D=-4.17 \text { (systolic BP) } \\
M D=-3.62 \text { (diastolic BP) } \\
\text { Yoga compared to usual care/no } \\
\text { treatment: } \\
M D=-7.96 \text { (systolic BP) } \\
M D=-5.52 \text { (diastolic BP) } \\
\text { Yoga compared to physical } \\
\text { exercise or active control: } \\
\text { No difference }\end{array}$ & $\begin{array}{l}\text { Significant reductions in } B P \\
\text { only in yoga interventions } \\
\text { that incorporated three } \\
\text { components of practice ( } \mathrm{P} \text {, } \\
\mathrm{B}, \mathrm{M} \text { ) (systolic: } M D=-8.17 \text {; } \\
\text { diastolic: } M D=-6.14 \text { ), and } \\
\text { not in interventions with } \\
\text { fewer components (MD = } \\
0.19 \text { n.s.; } M D=0.38 \text { n.s.). }\end{array}$ & $\begin{array}{l}\text { Combined } \\
\text { interventions are } \\
\text { more effective. }\end{array}$ \\
\hline
\end{tabular}




\begin{tabular}{|c|c|c|c|c|c|}
\hline Meta-analysis & Population/outcome & Sample & Mean effects & Subgroup analyses & Conclusion \\
\hline Hendriks et al. [8] & $\begin{array}{l}\text { Healthy } \\
\text { adults/indicators of } \\
\text { positive mental health }\end{array}$ & $\begin{array}{l}17 \text { RCTs } \\
(n=1,901)\end{array}$ & $\begin{array}{l}\text { Yoga compared to no treatment: } \\
\text { SMD }=0.69 \text { (well-being) } \\
\text { No effects on life satisfaction, } \\
\text { social relationships, or } \\
\text { mindfulness } \\
\text { Yoga compared to physical } \\
\text { exercise: } \\
\text { No difference }\end{array}$ & $\begin{array}{l}\text { The positive effect of yoga } \\
\text { on well-being was } \\
\text { comparable to the effects of } \\
\text { meditation or physical } \\
\text { exercise; mindfulness } \\
\text { meditation had a greater } \\
\text { effect on mindfulness } \\
\text { compared to yoga (SMD } \\
=-0.30 \text { ) }\end{array}$ & $\begin{array}{l}\text { No difference } \\
\text { between yoga and } \\
\text { meditation. }\end{array}$ \\
\hline Jiang et al. [72] & $\begin{array}{l}\text { People living with } \\
\text { HIV/immune function, } \\
\text { mental health }\end{array}$ & $\begin{array}{l}19 \text { RCTs } \\
(n=1,300)\end{array}$ & $\begin{array}{l}\text { Yoga and mindfulness-based } \\
\text { interventions } \\
\begin{array}{l}d=0.21 \text { (CD4 T-cell counts) } \\
d=0.43 \text { (long-term CD4) } \\
d=0.51 \text { (depression) } \\
d=0.71 \text { (anxiety) } \\
d=0.42 \text { (stress/distress) } \\
d=0.67 \text { (quality of life) }\end{array}\end{array}$ & $\begin{array}{l}\text { No significant differences } \\
\text { between yoga and } \\
\text { mindfulness-based } \\
\text { interventions on any } \\
\text { outcome variable. }\end{array}$ & $\begin{array}{l}\text { No differences } \\
\text { between complex } \\
\text { interventions. }\end{array}$ \\
\hline Kumar et al. [73] & $\begin{array}{l}\text { Type II diabetes } \\
\text { mellitus/glycemic } \\
\text { control }\end{array}$ & $\begin{array}{l}17 \text { RCTs } \\
(n=1,358)\end{array}$ & $\begin{array}{l}\text { Yoga compared to control: } \\
S M D=-1.40 \text { (fasting blood sugar) } \\
S M D=-0.91 \text { (postprandial blood } \\
\text { sugar) } \\
S M D=-0.64 \text { (glycosylated } \\
\text { hemoglobin) }\end{array}$ & $\begin{array}{l}\text { In contrast to the overall } \\
\text { positive effect, a subgroup } \\
\text { of breathing-based } \\
\text { interventions showed no } \\
\text { effect on any of the three } \\
\text { outcome measures (fasting: } \\
S M D=-0.62 \text { n.s.; post- } \\
\text { prandial: } S M D=-0.49 \text { n.s.; } \\
\text { hemoglobin: } S M D=-0.05 \\
\text { n.s.). }\end{array}$ & $\begin{array}{l}\text { Combined } \\
\text { interventions are } \\
\text { more effective. }\end{array}$ \\
\hline
\end{tabular}




\begin{tabular}{|c|c|c|c|c|c|}
\hline Meta-analysis & Population/outcome & Sample & Mean effects & Subgroup analyses & Conclusion \\
\hline Park \& Han [74] & $\begin{array}{l}\text { Hypertension/blood } \\
\text { pressure }\end{array}$ & $\begin{array}{l}13 \text { RCTs } \\
(n=753)\end{array}$ & $\begin{array}{l}\text { Yoga and meditation (MBSR and } \\
\text { TM) } \\
\text { Yoga: } \\
M D=-4.59 \text { (systolic BP) } \\
M D=-3.65 \text { (diastolic BP) } \\
\text { Meditation: } \\
M D=-7.37 \text { (systolic BP) } \\
M D=-5.43 \text { (diastolic BP) }\end{array}$ & $\begin{array}{l}\text { No significant differences } \\
\text { between yoga and } \\
\text { (combined) meditation } \\
\text { interventions. }\end{array}$ & $\begin{array}{l}\text { No differences } \\
\text { between } \\
\text { combined } \\
\text { interventions. }\end{array}$ \\
\hline Pascoe et al. [6] & $\begin{array}{l}\text { Any population/stress- } \\
\text { related physiological } \\
\text { measures }\end{array}$ & $\begin{array}{l}42 \text { RCTs } \\
(n=2,944)\end{array}$ & $\begin{array}{l}\text { Yoga and } M B S R \\
\text { Compared to active controls: } \\
M D=-1.51 \text { (waking cortisol) } \\
M D=-0.60 \text { (afternoon cortisol) } \\
M D=-0.88 \text { (evening cortisol) } \\
M D=-3.66 \text { (resting diastolic BP) } \\
M D=-6.82 \text { (resting mean arterial } \\
\text { pressure) } \\
M D=-3.20 \text { (resting heart rate) } \\
\text { Positive effects also on heart-rate } \\
\text { variability, fasting blood sugar, } \\
\text { cholesterol, low-density } \\
\text { lipoprotein } \\
\text { No effects on other measures }\end{array}$ & $\begin{array}{l}\text { Positive effects of MBSR } \\
\text { over yoga on decreasing } \\
\text { interleukin-6 (SMD = }-0.48 \\
\text { vs. SMD = }-0.10 \text { ) and of } \\
\text { yoga over MBSR on systolic } \\
\text { blood pressure (n.r.); both } \\
\text { types of interventions } \\
\text { reduced salivary cortisol, } \\
\text { BP, and cytokine levels to a } \\
\text { greater extent than active } \\
\text { controls }\end{array}$ & $\begin{array}{l}\text { No differences } \\
\text { between } \\
\text { combined } \\
\text { interventions }\end{array}$ \\
\hline Taylor et al. [75] & $\begin{array}{l}\text { Adults with trauma } \\
\text { exposure/trauma- } \\
\text { related symptoms } \\
\text { (PTSD, stress, anxiety) }\end{array}$ & $\begin{array}{l}24 \text { controlled } \\
\text { studies } \\
(n=759)\end{array}$ & $\begin{array}{l}\text { Yoga, mindfulness/MBSR, and } \\
\text { integrative exercise } \\
g=0.48 \text { (overall pooled effect) } \\
g=0.46 \text { (overall - yoga) } \\
g=0.45 \text { (overall - mindfulness) }\end{array}$ & $\begin{array}{l}\text { No differences between } \\
\text { mindfulness-based and } \\
\text { yoga interventions with at } \\
\text { least two limbs of yoga. }\end{array}$ & $\begin{array}{l}\text { No differences } \\
\text { between } \\
\text { combined } \\
\text { interventions. }\end{array}$ \\
\hline
\end{tabular}




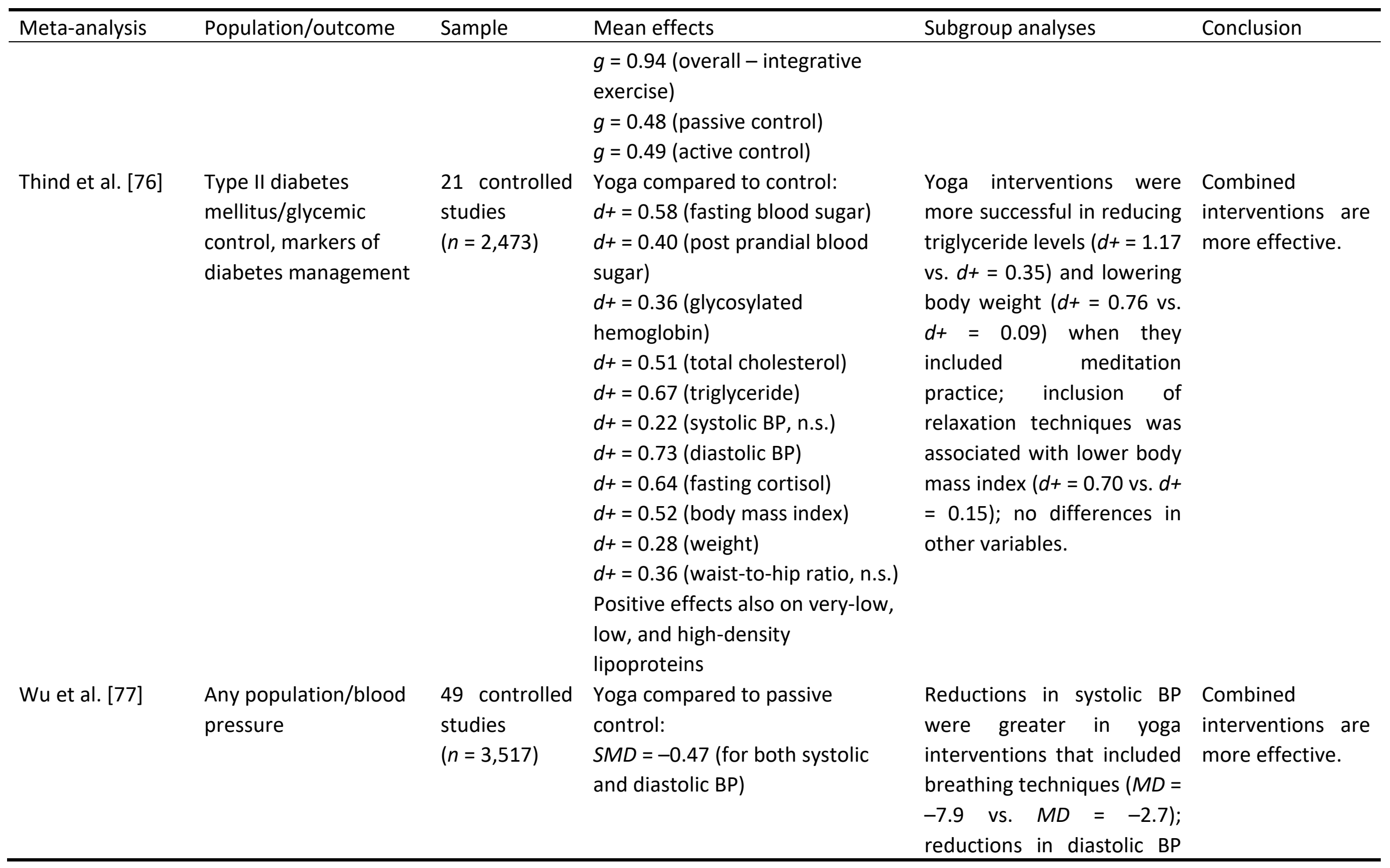




\begin{tabular}{|c|c|c|c|c|c|}
\hline Meta-analysis & Population/outcome & Sample & Mean effects & Subgroup analyses & Conclusion \\
\hline & & & & $\begin{array}{l}\text { were greater in yoga } \\
\text { interventions that included } \\
\text { meditation/mental } \\
\text { relaxation (MD = -4.3 vs. } \\
M D=-1.6) .\end{array}$ & \\
\hline
\end{tabular}

Note. $\mathrm{ADHD}=$ attention-deficit/hyperactivity disorder; $\mathrm{B}=$ breathing; $\mathrm{BP}=$ blood pressure $\mathrm{COPD}=$ chronic obstructive pulmonary disease; $d+=$ weighted mean effect size with positive values favoring the intervention relative to control; $g=$ Hedges' $g ; M=$ meditation; $M B C T=$ mindfulness-based cognitive therapy; $\mathrm{MBSR}=$ mindfulness-based stress reduction; $M D=$ mean difference; $\mathrm{n} . \mathrm{r}$. = not reported; $\mathrm{P}=$ postures; $\mathrm{R}=$ relaxation; $S M D=$ standardized mean difference; $T M=$ Transcendental Meditation. Reported effects are significant unless otherwise stated. 
The meta-analyses summarized in Table 1 are very diverse. The majority of them investigated clinical populations with conditions such as depression, hypertension, chronic pain, asthma, chronic obstructive pulmonary disease (COPD), inflammatory bowel disease, type II diabetes, HIV, trauma, and attention-deficit/hyperactivity disorder. Three meta-analyses used mixed samples, including healthy and clinical populations, and examined the effect of yoga on cognition [70], stress-related physiological measures [6], and the prevention of and therapy for hypertension [77]. Only two metaanalyses investigated healthy populations. One of them looked at depression, anxiety, and stress in college students [5], and the other explored positive mental health [8]. The latter concluded that there was a scarcity of studies investigating the effects of yoga on healthy populations, and specifically those measuring positive outcomes. This is surprising as yoga initially was developed as a spiritual path for healthy people and not as a treatment for clinical conditions [78].

The majority of the meta-analyses reported favorable effects of yoga (and other mind-body interventions) on depression, anxiety, stress, and stress-related physiological measures, well-being, mood, quality of life, cognitive function, and several other clinical symptoms. Most effects were small to moderate and usually large when yoga was compared with passive control or usual care. Compared with active control conditions, yoga/mind-body interventions exerted a moderate effect on several physiological measures [6], trauma symptoms [75], and levels of depression in clinical samples $[66,69]$. However, yoga's positive effects diminished compared to physical exercise or other active controls in healthy populations $[5,8]$ and in populations with asthma [67] or hypertension $[64,71]$.

In an earlier summary of reviews, Büssing et al. [7] concluded that yoga postures could be particularly beneficial for fitness, physical flexibility, and mental state. In contrast, breathing, relaxation, and meditation techniques could lead to greater awareness, less stress, and higher wellbeing and quality of life. This conclusion might also apply to the more recent meta-analyses displayed in Table 1.

A majority of the meta-analyses reported that combined interventions led to moderately higher effects than simpler yoga interventions or yoga postures $[62,66,67,69-71,73,76,77]$. Sometimes, this implied that positive results were only obtained for interventions that included specific yoga components and were absent in other interventions that did not $[68,76]$. In other instances, differences between interventions were small, but significant for one specific component or for one combination and insignificant for others $[64,66,69,71,73]$. Combined interventions appeared to be beneficial, specifically for clinical samples. Breathing and meditation techniques were particularly advantageous in this regard, and interventions based on these practices were more effective than yoga postures in increasing cognitive performance [70], reducing depressive symptoms [66], and downregulating blood pressure [64]. Similarly, including breathing and/or meditation practices in yoga interventions increased their effectiveness in reducing prenatal depression [69] and elevated blood pressure $[71,77]$. Unsurprisingly, yoga interventions based on or including breathing practices were particularly helpful in alleviating respiratory diseases, such as asthma [67] and COPD [63]. In contrast, breathing-based yoga was not as effective in improving glycemic control and other diabetes-related measures $[73,76]$. The latter meta-analysis found that including meditation or relaxation practices was more helpful in this respect. No differences were found between exercisebased and meditation-based yoga interventions in alleviating chronic neck pain [65].

Consequently, specific clinical conditions or objectives could require specific combinations of yoga practices to elicit the highest benefits. Future research should examine these combinations in 
more detail and evaluate what combinations apply to a given condition/objective. This could eventually lead to the development of programs tailored to the needs of specific populations [17, $18,79]$.

Meta-analyses on healthy populations reported no differences between meditation, mindfulness-based, or yoga interventions $[5,8]$. Similarly, no differences were observed between these interventions regarding hypertension [74], trauma symptoms [75], stress-related physiological measures [6], and immunological outcomes and mental health of people living with HIV [72]. However, these meta-analyses did not differentiate the components of yoga or mindfulness-based practices and are, thus, less informative than other meta-analyses that do. However, these meta-analyses suggest that equally complex interventions such as yoga and mindfulness-based treatments yield similar effects. Conversely, MBSR was more effective than yoga in reducing hyperactivity [62], and yoga was more effective than MBSR in decreasing depression and anxiety in patients with inflammatory bowel disease [68]. Thus, the findings are inconclusive. More research is required to determine the unique contribution of each component to the globally positive effect of combined interventions.

\section{Discussion}

Overall, the yoga studies and meta-analyses reviewed above highly vary with regard to the population, yoga components under investigation, intervention length, and control intervention(s). This appears to be symptomatic of yoga research, in general $[80,81]$. To increase the transparency and replicability of intervention studies, an extensive framework for developing yoga treatment protocols has been proposed and recommended [82]. However, a systematic empirical investigation into the various components of yoga practice is missing.

One finding that was consistent throughout the reviewed literature was the superiority of combined over simple interventions. Treatments that included more components of traditional yoga, such as ethical education, postures, breathing, or meditation, were more effective than those that were based on fewer components. Particularly, breathing and meditation practices enhanced the efficacy of yoga treatments. However, certain combinations of yoga practices were more useful for specific study populations or outcome variables than others-which combinations exactly has to be determined in future studies as the available evidence on this matter is still sparse.

None of the meta-analyses we reviewed examined the additive value of including the ethical aspect of yoga in intervention studies. Similarly, only a few comparative investigations explicitly studied the effects of (ethical) education. This is one of the major shortcomings of previous yoga research. The idea of integrating more philosophical or ethical components into mind-body interventions has recently been discussed in the mindfulness literature [83-86]. Chen and Jordan [87] compared an ethically enriched intervention with a standard mindfulness intervention and reported that both interventions reduced stress and increased life satisfaction. However, the ethical intervention also enhanced personal growth and prosocial behavior. Similar results were obtained in the abovementioned ethical yoga studies [34, 39]. Moreover, recent theoretical proposals [15, 20] suggest evaluating yoga holistically, including its ethical aspects. Therefore, it is essential to study the impact of ethical education on the outcomes of yoga treatments. The recently developed mind-body intervention meditation-based lifestyle modification [88] could be useful in this regard as it explicitly incorporates lectures on yogic yamas and niyamas. 
Our review suggests that comparable multi-component mind-body interventions, such as yoga and mindfulness-based interventions, produce similar effects. Although the two types of interventions originate from different spiritual traditions (Hinduism and Buddhism, respectively), they share similar exercises. Both yoga and MBSR (but not necessarily other mindfulness-based interventions) include yoga postures, relaxation, meditation, and an educational component. Yet, the yoga posture component of MBSR treatments is rather basic compared to most yoga interventions. Recently, two studies dismantled MBSR treatments and found differential effects of its components $[13,58,59]$. Nevertheless, more research is necessary to thoroughly understand how each component and also the relative emphasis placed on each component within MBSR or yoga contribute to the efficacy of combined treatments. In addition, current research has demonstrated that Hindu and Buddhist meditators had extremely distinct preferences for specific meditation techniques [89]. These differences between traditions might ultimately influence the outcomes of both related interventions and account for the observed differences reviewed above.

To disentangle the components of yoga and other mind-body interventions, future studies should aim at either comparing specific, isolated components to each other or using additive designs to evaluate the incremental effects of different combinations of yoga practices. Moreover, it is essential to evaluate yoga in its entirety, including postures, breathing, meditation, and ethics. This is particularly relevant in the light of the undeniable diversity of yoga studies and interventions reviewed above. To understand the basic working mechanisms of yoga, comparative studies should rely on healthy populations and aim for representative samples. In contrast, clinical studies could evaluate diverse combinations of yoga practices to find those most suited to specific clinical conditions. Furthermore, long-term effects should be considered. For example, Cramer et al. [12] showed that although a yoga intervention without postures was immediately more effective in reducing hypertension than an intervention with postures, the effect reversed in long-term followup. The authors argue that more participants continued to practice yoga postures regularly after the intervention had ended. Yoga postures might, thus, be easier to integrate into participants' daily activities.

Future studies should also take personality factors into account, as several authors have pointed out that individual differences could tremendously influence the effects of yoga and other mindbody interventions $[15,18,90]$. Researchers could achieve this by using more elaborate research designs offering a higher time resolution and keeping a better track of individual responses. Singlecase research designs [91] or ecological momentary assessment [92] appear to be promising approaches in this regard.

The majority of studies reviewed here investigated the effects of yoga on depression, anxiety, stress, well-being, and mood. Although yoga interventions, particularly the combined ones, commonly yielded positive effects on these variables, future studies should focus more on positive outcome variables [8]. This could lead to a holistic understanding of yoga and move the discipline away from a deficiency-oriented and toward a more flourishing-oriented perspective. Furthermore, several variables in the previous research were selected ad hoc without referring to specific theoretical frameworks. This is another issue that is symptomatic of yoga research $[15,18]$. Accordingly, future studies should compare not only different yoga components but also base these comparisons and the selection of appropriate variables on existing traditional or contemporary theoretical proposals. 
There are a couple of limitations to the findings of this meta-synthesis. First, the quality of studies differed widely within and between the meta-analyses reviewed. Although most meta-analyses included only RCTs, others were more liberal regarding study design, increasing the risk of biased or overestimated results. In addition, not all meta-analyses differentiated between different types of control conditions, making it difficult to achieve reliable conclusions. However, this was not the primary focus of this synthesis as we were interested majorly in studying the differential effects of yoga components. Second, certain meta-analyses could have had some overlap between included studies, particularly the several meta-analyses on hypertension reviewed here. Nevertheless, conclusions varied across these meta-analyses, reinforcing the need for more systematic research. Third, subgroup analyses differed between meta-analyses with certain comparing rather broad categories of mind-body interventions. Most meta-analyses differentiated between interventions, including postures, breathing and/or meditation practices, or combinations of the three; however, which subgroups exactly were compared to each other differed widely. Several meta-analyses we screened during the review process stated that they had planned to conduct subgroup analyses on yoga components. Yet, these were mostly not able to do so because of insufficient studies available or yoga interventions not described in enough detail. Consequently, future research would benefit greatly from a more systematic approach and more detailed descriptions of interventions.

Overall, there exists a high variability and ambiguity in yoga research with repeated calls to investigate differential effects of yoga components systematically. One particularly underresearched area is the incorporation of yoga ethics into intervention studies. We encourage researchers to use rigorous and elaborate research designs to examine all components of yoga, individually and in diverse combinations. In the end, these research efforts could contribute substantially to a deep understanding of the manifold effects of the multifaceted practice of yoga.

\section{Acknowledgements}

We thank Annika Sternkopf and Nele Boll for their help in gathering and screening the literature. Thank you to Anita Todd and two anonymous reviewers for their valuable comments on a previous version of this manuscript.

\section{Author Contributions}

KM designed and executed the meta-synthesis and wrote the paper. PS and HCB collaborated on the editing process. All authors approved the final version of the manuscript.

\section{Funding}

This work was supported by Karl and Veronica Carstens Foundation under Grant Number KVC 0/098/2018.

\section{Competing Interests}

The authors declare that no competing interests exist. 


\section{References}

1. Clarke TC, Black LI, Stussman BJ, Barnes PM, Nahin RL. Trends in the use of complementary health approaches among adults: United States, 2002-2012. Natl Health Stat Rep. 2015: 1-16. PMID: 25671660.

2. Eardley S, Bishop FL, Prescott P, Cardini F, Brinkhaus B, Santos-Rey K, et al. A systematic literature review of complementary and alternative medicine prevalence in EU. Complement Med Res. 2012; 19: 18-28.

3. Park CL, Riley KE, Bedesin E, Stewart VM. Why practice yoga? Practitioners' motivations for adopting and maintaining yoga practice. J Health Psychol. 2016; 21: 887-896.

4. Park CL, Quinker D, Dobos G, Cramer H. Motivations for adopting and maintaining a yoga practice: A national cross-sectional survey. J Altern Complement Med. 2019; 25: 1009-1014.

5. Breedvelt JJ, Amanvermez Y, Harrer M, Karyotaki E, Gilbody S, Bockting CL, et al. The effects of meditation, yoga, and mindfulness on depression, anxiety, and stress in tertiary education students: A meta-analysis. Front Psychiatry. 2019; 10: 1-15.

6. Pascoe MC, Thompson DR, Ski CF. Yoga, mindfulness-based stress reduction and stress-related physiological measures: A meta-analysis. Psychoneuroendocrinology. 2017; 86: 152-168.

7. Büssing A, Michalsen A, Khalsa SB, Telles S, Sherman KJ. Effects of yoga on mental and physical health: A short summary of reviews. Evid Based Complementary Altern Med. 2012; 2012: 1-7.

8. Hendriks $\mathrm{T}$, de Jong J, Cramer $\mathrm{H}$. The effects of yoga on positive mental health among healthy adults: A systematic review and meta-analysis. J Altern Complement Med. 2017; 23: 505-517.

9. Ross A, Thomas S. The health benefits of yoga and exercise: A review of comparison studies. J Altern Complement Med. 2010; 16: 3-12.

10. Feuerstein G. The yoga tradition: Its history, literature, philosophy and practice. Chino Valley, Arizona: Hohm Press; 2012.

11. Telles $S$, Singh N. Science of the mind: Ancient yoga texts and modern studies. Psychiatr Clin. 2013; 36: 93-108.

12. Cramer H, Sellin C, Schumann D, Dobos G. Yoga in arterial hypertension: A three-armed, randomized controlled trial. Dtsch Arztebl Int. 2018; 115: 833-839.

13. Sauer-Zavala SE, Walsh EC, Eisenlohr-Moul TA, Lykins EL. Comparing mindfulness-based intervention strategies: Differential effects of sitting meditation, body scan, and mindful yoga. Mindfulness. 2013; 4: 383-388.

14. Trakroo M, Bhavanani AB, Pal GK, Udupa K, Krishnamurthy N. A comparative study of the effects of asan, pranayama and asan-pranayama training on neurological and neuromuscular functions of Pondicherry police trainees. Int J Yoga. 2013; 6: 96-103.

15. Gard T, Noggle JJ, Park CL, Vago DR, Wilson A. Potential self-regulatory mechanisms of yoga for psychological health. Front Hum Neurosci. 2014; 8: 1-20.

16. McCall MC. How might yoga work? An overview of potential underlying mechanisms. J yoga phys ther. 2013; 3: 1-6.

17. Riley KE, Park CL. How does yoga reduce stress? A systematic review of mechanisms of change and guide to future inquiry. Health Psychol Rev. 2015; 9: 379-396.

18. SchmalzI L, Powers C, Blom EH. Neurophysiological and neurocognitive mechanisms underlying the effects of yoga-based practices: Towards a comprehensive theoretical framework. Front Hum Neurosci. 2015; 9: 1-19. 
19. Matko K, Sedlmeier P. What is meditation? Proposing an empirically derived classification system. Front Psychol. 2019; 10: 1-14.

20. Sullivan MB, Moonaz S, Weber K, Taylor JN, Schmalzl L. Toward an explanatory framework for yoga therapy informed by philosophical and ethical perspectives. Altern Ther Health Med. 2017; 23: 251-259.

21. McCrary M. Pick your yoga practice: Exploring and understanding different styles of yoga. Novato, California: New World Library; 2013.

22. Peng CK, Henry IC, Mietus JE, Hausdorff JM, Khalsa G, Benson $H$, et al. Heart rate dynamics during three forms of meditation. Int J Cardiol. 2004; 95: 19-27.

23. Raghuraj P, Ramakrishnan AG, Nagendra HR, Telles S. Effect of two selected yogic breathing techniques on heart rate variability. Indian J Physiol Pharmacol. 1998; 42: 467-472.

24. Quach D, Mano KE, Alexander K. A randomized controlled trial examining the effect of mindfulness meditation on working memory capacity in adolescents. J Adolesc Health. 2016; 58: 489-496.

25. Smith C, Hancock H, Blake-Mortimer J, Eckert K. A randomised comparative trial of yoga and relaxation to reduce stress and anxiety. Complement Ther Med. 2007; 15: 77-83.

26. Granath J, Ingvarsson S, von Thiele U, Lundberg U. Stress management: A randomized study of cognitive behavioural therapy and yoga. Cogn Behav Ther. 2006; 35: 3-10.

27. Park CL, Riley KE, Braun TD, Jung JY, Suh HG, Pescatello LS, et al. Yoga and cognitive-behavioral interventions to reduce stress in incoming college students: A pilot study. J Appl Biobehav Res. 2017; 22: e12068.

28. Qi X, Tong J, Chen S, He Z, Zhu X. Comparing the psychological effects of meditation-and breathing-focused yoga practice in undergraduate students. Front Psychol. 2020; 11: 560152.

29. Saksena T, Sharma R, Basavaraddi IV. Exploring the role of Hatha yoga in altering dispositional mindfulness. Indian J Public Health Res Dev. 2020; 11: 868-873.

30. SchmalzI L, Powers C, Zanesco AP, Yetz N, GroessI EJ, Saron CD. The effect of movement-focused and breath-focused yoga practice on stress parameters and sustained attention: A randomized controlled pilot study. Conscious Cogn. 2018; 65: 109-125.

31. Melville GW, Chang D, Colagiuri B, Marshall PW, Cheema BS. Fifteen minutes of chair-based yoga postures or guided meditation performed in the office can elicit a relaxation response. Evid Based Complementary Altern Med. 2012; 2012: 1-9.

32. Wheeler EA, Santoro AN, Bembenek AF. Separating the "limbs" of yoga: Limited effects on stress and mood. J Relig Health. 2019; 58: 2277-2287.

33. Bowden D, Gaudry C, An SC, Gruzelier J. A comparative randomised controlled trial of the effects of Brain Wave Vibration Training, lyengar Yoga, and mindfulness on mood, well-being, and salivary cortisol. Evid Based Complementary Altern Med. 2011; 2012: 1-13.

34. Matko K, Sedlmeier P, Bringmann HC. Differential effects of ethical education, physical Hatha yoga, and mantra meditation on well-being and stress in healthy participants - an experimental single-case study. Front Psychol. 2021; 12: 3050.

35. Rohini V, Pandey RS, Janakiramaiah N, Gangadhar BN, Vedamurthachar A. A comparative study of full and partial Sudarshan Kriya Yoga (SKY) in major depressive disorder. NIMHANS J. 2000; 18: 53-57.

36. Shankarapillai R, Nair MA, George R. The effect of yoga in stress reduction for dental students performing their first periodontal surgery: A randomized controlled study. Int J Yoga. 2012; 5: 
48-51.

37. Shannahoff-Khalsa DS, Ray LE, Levine S, Gallen CC, Schwartz BJ, Sidorowich JJ. Randomized controlled trial of yogic meditation techniques for patients with obsessive-compulsive disorder. CNS Spectr. 1999; 4: 34-47.

38. Shannahoff-Khalsa D, Fernandes RY, de B Pereira CA, March JS, Leckman JF, Golshan S, et al. Kundalini Yoga meditation versus the Relaxation Response meditation for treating adults with obsessive-compulsive disorder: A randomized clinical trial. Front Psychiatry. 2019; 10: 1-15.

39. Smith JA, Greer T, Sheets T, Watson S. Is there more to yoga than exercise? Altern Ther Health Med. 2011; 17: 22-29.

40. Telles S, Gaur V, Balkrishna A. Effect of a yoga practice session and a yoga theory session on state anxiety. Percept Mot Skills. 2009; 109: 924-930.

41. Wolever RQ, Bobinet KJ, McCabe K, Mackenzie ER, Fekete E, Kusnick CA, et al. Effective and viable mind-body stress reduction in the workplace: A randomized controlled trial. J Occup Health Psychol. 2012; 17: 246-258.

42. Falsafi N. A randomized controlled trial of mindfulness versus yoga: Effects on depression and/or anxiety in college students. J Am Psychiatr Nurses Assoc. 2016; 22: 483-497.

43. Gorvine MM, Zaller ND, Hudson HK, Demers D, Kennedy LA. A naturalistic study of yoga, meditation, self-perceived stress, self-compassion, and mindfulness in college students. Health Psychol Behav Med. 2019; 7: 385-395.

44. Bhat PS, Chopra V, Mehta SG, Srivastava K, Kumar SR, Prakash J. Psychological benefits of yoga in industrial workers. Ind Psychiatry J. 2012; 21: 98-103.

45. Bragard I, Etienne AM, Faymonville ME, Coucke P, Lifrange E, Schroeder $H$, et al. A nonrandomized comparison study of self-hypnosis, yoga, and cognitive-behavioral therapy to reduce emotional distress in breast cancer patients. Int J Clin Exp Hypn. 2017; 65: 189-209.

46. Simon NM, Hofmann SG, Rosenfield D, Hoeppner SS, Hoge EA, Bui E, et al. Efficacy of yoga vs cognitive behavioral therapy vs stress education for the treatment of generalized anxiety disorder: A randomized clinical trial. JAMA psychiatry. 2021; 78: 13-20.

47. Bhavanani AB, Ramanathan M, Dayanidy G, Trakroo M, Renuka K. A comparative study of the differential effects of short term asana and pranayama training on reaction time. Ann Med Health Sci Res. 2017; 7: 80-83.

48. Vasanthan $S$, Madanmohan $T$, Bhavanani AB, Hanifah M, Jaiganesh K. Comparative study on the effect of yogic relaxing asanas and pranayamas on cardiovascular response in healthy young volunteers. Natl J Physiol Pharm Pharmacol. 2017; 7: 127-130.

49. Brown RP, Gerbarg PL. Sudarshan Kriya yogic breathing in the treatment of stress, anxiety, and depression: Part I-neurophysiologic model. J Altern Complement Med. 2005; 11: 189-201.

50. Bhogal RS, Thakur GS, Shete SU. Differential impact of Shavasana and meditation on memory scores in healthy college students: A randomized controlled study. Yoga Mimamsa. 2016; 48: 9-12.

51. Varambally S, Gangadhar BN. Current status of yoga in mental health services. Int Rev Psychiatry. 2016; 28: 233-235.

52. de Manincor M, Bensoussan A, Smith C, Fahey P, Bourchier S. Establishing key components of yoga interventions for reducing depression and anxiety, and improving well-being: A Delphi method study. BMC Complement Altern Med. 2015; 15: 1-10.

53. Park CL, Elwy AR, Maiya M, Sarkin AJ, Riley KE, Eisen SV, et al. The essential properties of yoga 
questionnaire (EPYQ): Psychometric properties. Int J Yoga Ther. 2018; 28: 23-38.

54. Kabat-Zinn J. An outpatient program in behavioral medicine for chronic pain patients based on the practice of mindfulness meditation: Theoretical considerations and preliminary results. Gen Hosp Psychiatry. 1982; 4: 33-47.

55. Carmody J, Baer RA. Relationships between mindfulness practice and levels of mindfulness, medical and psychological symptoms and well-being in a mindfulness-based stress reduction program. J Behav Med. 2008; 31: 23-33.

56. Gallegos AM, Hoerger M, Talbot NL, Krasner MS, Knight JM, Moynihan JA, et al. Toward identifying the effects of the specific components of Mindfulness-Based Stress Reduction on biologic and emotional outcomes among older adults. J Altern Complement Med. 2013; 19: 787-792.

57. Colgan DD, Christopher M, Michael $\mathrm{P}$, Wahbeh $\mathrm{H}$. The body scan and mindful breathing among veterans with PTSD: Type of intervention moderates the relationship between changes in mindfulness and post-treatment depression. Mindfulness. 2016; 7: 372-383.

58. Hunt M, Al-Braiki F, Dailey S, Russell R, Simon K. Mindfulness training, yoga, or both? Dismantling the active components of a mindfulness-based stress reduction intervention. Mindfulness. 2018; 9: 512-520.

59. Kropp A, Sedlmeier P. What makes mindfulness-based interventions effective? An examination of common components. Mindfulness. 2019; 10: 2060-2072.

60. Stein $\mathrm{E}$, Witkiewitz K. Dismantling mindfulness-based programs: A systematic review to identify active components of treatment. Mindfulness. 2020; 11: 2470-2485.

61. Cooper $\mathrm{H}$, Koenka AC. The overview of reviews: Unique challenges and opportunities when research syntheses are the principal elements of new integrative scholarship. Am Psychol. 2012; 67: 446-462.

62. Chimiklis AL, Dahl V, Spears AP, Goss K, Fogarty K, Chacko A. Yoga, mindfulness, and meditation interventions for youth with ADHD: Systematic review and meta-analysis. J Child Fam Stud. 2018; 27: 3155-3168.

63. Cramer H, Haller H, Klose P, Ward L, Chung VC, Lauche R. The risks and benefits of yoga for patients with chronic obstructive pulmonary disease: A systematic review and meta-analysis. Clin Rehabil. 2019; 33: 1847-1862.

64. Cramer H, Haller H, Lauche R, Steckhan N, Michalsen A, Dobos G. A systematic review and metaanalysis of yoga for hypertension. Am J Hypertens. 2014; 27: 1146-1151.

65. Cramer H, Klose $P$, Brinkhaus B, Michalsen A, Dobos G. Effects of yoga on chronic neck pain: A systematic review and meta-analysis. Clin Rehabil. 2017; 31: 1457-1465.

66. Cramer H, Lauche R, Langhorst J, Dobos G. Yoga for depression: A systematic review and metaanalysis. Depress Anxiety. 2013; 30: 1068-1083.

67. Cramer H, Posadzki P, Dobos G, Langhorst J. Yoga for asthma: A systematic review and metaanalysis. Ann Allergy Asthma Immunol. 2014; 112: 503-510.e5.

68. Ewais T, Begun J, Kenny M, Rickett K, Hay K, Ajilchi B, et al. A systematic review and metaanalysis of mindfulness based interventions and yoga in inflammatory bowel disease. $J$ Psychosom Res. 2019; 116: 44-53.

69. Gong $\mathrm{H}, \mathrm{Ni}$ C, Shen $\mathrm{X}, \mathrm{Wu} \mathrm{T}$, Jiang $\mathrm{C}$. Yoga for prenatal depression: A systematic review and meta-analysis. BMC Psychiatry. 2015; 15: 1-8.

70. Gothe NP, McAuley E. Yoga and cognition: A meta-analysis of chronic and acute effects. 
Psychosom Med. 2015; 77: 784-797.

71. Hagins M, States R, Selfe T, Innes K. Effectiveness of yoga for hypertension: Systematic review and meta-analysis. Evid Based Complementary Altern Med. 2013; 2013: 1-13.

72. Jiang $T$, Hou J, Sun R, Dai L, Wang W, Wu H, et al. Immunological and psychological efficacy of meditation/yoga intervention among people living with HIV (PLWH): A systematic review and meta-analyses of 19 randomized controlled trials. Ann Behav Med. 2021; 55: 505-519.

73. Kumar V, Jagannathan A, Philip M, Thulasi A, Angadi P, Raghuram N. Role of yoga for patients with type II diabetes mellitus: A systematic review and meta-analysis. Complement Ther Med. 2016; 25: 104-112.

74. Park S-H, Han KS. Blood pressure response to meditation and yoga: A systematic review and meta-analysis. J Altern Complement Med. 2017; 23: 685-695.

75. Taylor J, McLean L, Korner A, Stratton E, Glozier N. Mindfulness and yoga for psychological trauma: Systematic review and meta-analysis. J Trauma Dissociation. 2020; 21: 536-573.

76. Thind $H$, Lantini $R$, Balletto BL, Donahue ML, Salmoirago-Blotcher E, Bock BC, et al. The effects of yoga among adults with type 2 diabetes: A systematic review and meta-analysis. Prev Med. 2017; 105: 116-126.

77. Wu Y, Johnson BT, Acabchuk RL, Chen S, Lewis HK, Livingston J, et al. Yoga as antihypertensive lifestyle therapy: A systematic review and meta-analysis. Mayo Clin Proc. 2019; 94: 432-46.

78. Sedlmeier P, Srinivas K. Psychological theories of meditation based on early Buddhism and Samkhya/Yoga. In: The Oxford handbook of meditation. Oxford: Oxford University Press; 2019.

79. Gard T, Brach N, Hölzel BK, Noggle JJ, Conboy LA, Lazar SW. Effects of a yoga-based intervention for young adults on quality of life and perceived stress: The potential mediating roles of mindfulness and self-compassion. J Posit Psychol. 2012; 7: 165-175.

80. Cramer $H$, Lauche $R$, Dobos $G$. Characteristics of randomized controlled trials of yoga: $A$ bibliometric analysis. BMC Complement Altern Med. 2014; 14: 1-20.

81. Elwy AR, Groessl EJ, Eisen SV, Riley KE, Maiya M, Lee JP, et al. A systematic scoping review of yoga intervention components and study quality. Am J Prev Med. 2014; 47: 220-232.

82. Sherman KJ. Guidelines for developing yoga interventions for randomized trials. Evid Based Complementary Altern Med. 2012; 2012: 1-16.

83. Baer R. Ethics, values, virtues, and character strengths in mindfulness-based interventions: A psychological science perspective. Mindfulness. 2015; 6: 956-969.

84. Gordon WV, Shonin E, Griffiths MD. Towards a second generation of mindfulness-based interventions. Aust N Z J Psychiatry. 2015; 49: 591-592.

85. Monteiro LM, Musten RF, Compson J. Traditional and contemporary mindfulness: Finding the middle path in the tangle of concerns. Mindfulness. 2015; 6: 1-13.

86. Purser RE. Clearing the muddled path of traditional and contemporary mindfulness: A response to Monteiro, Musten, and Compson. Mindfulness. 2015; 6: 23-45.

87. Chen $\mathrm{S}$, Jordan $\mathrm{CH}$. Incorporating ethics into brief mindfulness practice: Effects on well-being and prosocial behavior. Mindfulness. 2020; 11: 18-29.

88. Bringmann HC, Bringmann N, Jeitler M, Brunnhuber S, Michalsen A, Sedlmeier P. Meditationbased lifestyle modification: Development of an integrative mind-body program for mental health and human flourishing. Complement Med Res. 2021; 28: 252-262.

89. Matko K, Ott U, Sedlmeier P. What do meditators do when they meditate? Proposing a novel basis for future meditation research. Mindfulness. 2021; 12: 1791-1811. 
90. Hölzel BK, Lazar SW, Gard T, Schuman-Olivier Z, Vago DR, Ott U. How does mindfulness meditation work? Proposing mechanisms of action from a conceptual and neural perspective. Perspect Psychol Sci. 2011; 6: 537-559.

91. Barlow $\mathrm{DH}$, Nock $M$, Hersen $M$. Single case experimental designs: Strategies for studying behavior for change. 3rd ed. Boston, MA: Pearson; 2009.

92. Shiffman S, Stone AA, Hufford MR. Ecological momentary assessment. Annu Rev Psychol. 2008; 4: 1-32.

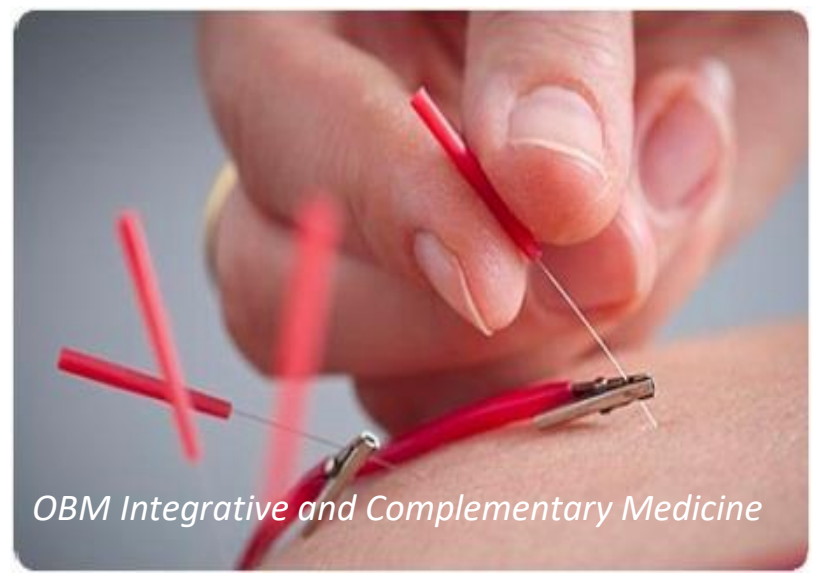

Enjoy OBM Integrative and Complementary Medicine by:

1. Submitting a manuscript

2. Joining in volunteer reviewer bank

3. Joining Editorial Board

4. Guest editing a special issue

For more details, please visit: http://www.lidsen.com/iournals/icm 